\title{
ANÁLISIS COSTE-UTILIDAD DE LAS BOMBAS DE INSULINA FRENTE A MÚLTIPLES DOSIS DIARIAS EN PACIENTES CON DIABETES MELLITUS TIPO 1 EN ESPAÑA (*)
}

\author{
Ignacio Conget Donlo (1), David Serrano Contreras (2), José Manuel Rodríguez Barrios (2), Isaac \\ Levy Mizrahi (1), Conxa Castell Abat (3) y Stephan Roze (4) \\ (1) Servicio de Endocrinología y Diabetes. Hospital Clínic i Universitari, IDIBAPS Barcelona. España. \\ (2) Economía de la Salud y Reembolso. Medtronic Ibérica. Madrid. España \\ (3) Consell Assessor sobre la Diabetis a Catalunya (CADC). Direcció General de Salut Pública. Departament de Salut. \\ Generalitat de Catalunya. España \\ (4) CORE - Center for Outcomes Research. Basilea. Suiza \\ (*) Tanto el desarrollo como la elaboración del modelo ha sido financiado por una beca no finalista otorgada por Med- \\ tronic Ibérica S.A. a CORE Research, quien ha llevado a cabo los análisis en todo momento y con total autonomía.
}

\section{RESUMEN}

Fundamento: El uso de bombas de infusión continua de insulina (BICI) para la diabetes mellitus tipo 1 (DM1) se ha relacionado con un mejor control metabólico al compararlo con las múltiples dosis de insulina (MDI). Este mejor control puede traducirse en una disminución de las complicaciones asociadas a la DM1 y por lo tanto una reducción de los costes asociados. Sin embargo el uso de esta terapia ha quedado mermado, al menos en parte, debido a su mayor coste inicial de adquisición. El objetivo del presente estudio fue estimar las consecuencias clínicas y económicas del uso de BICI frente a MDI a través de un análisis de coste-utilidad.

Métodos: Se adaptó un modelo matemático de simulación que emplea datos clínicos y económicos de ámbito nacional, para simular las consecuencias clínicas y económicas a largo plazo de un paciente con DM1. El horizonte temporal fue el de toda la vida del paciente, incluyendo sólo costes directos sanitarios, y actualizando tanto costes como beneficios a una tasa del $3 \%$ anual.

Resultados: En el caso base los pacientes tratados con BICI experimentaron una ganancia de vida de 0,890 años $(\mathrm{p}<0,05)$ y 0,852 AVACs $(\mathrm{p}<0,05)$. El tratamiento con BICI produce un coste medio incremental de $25.523 €(\mathrm{p}<0,05)$ por paciente tratado, lo que nos condujo a un ratio coste- utilidad incremental de $29.947 €$ $€$ AVAC [IC $95 \%(29.519,30.375)]$.

Conclusiones: La mejora en el control glucémico en pacientes con BICI se asoció a una reducción del coste global del manejo de pacientes con DM1, y resultó tener una relación coste-utilidad favorable al compararla con el tratamiento convencional MDI.

Palabras clave: Diabetes mellitus Tipo 1. Coste-utilidad. Bombas de insulina. Modelo Económico.

Correspondencia:

José Manuel Rodríguez

Economía de la Salud y Reembolso. Medtronic Ibérica.

C/ María de Portugal 11

28050 Madrid. España

Correo electrónico: jose_manuel.rodriguez@medtronic.com

\section{ABSTRACT \\ Cost-Utility Analysis of Iinsulin Pumps Compared to Multiple Daily doses of Insulin in Patients with Type 1 Diabetes Mellitus in Spain}

Background: The use of continuous subcutaneous insulin infusion (CSII) for treating Type I diabetes mellitus (DM1) has been related to better metabolic control compared it to daily multiple insulin injections (DMI) and thus to a lowering of the related costs. However, this therapy is now being used to a lesser extent due, at least partially, to the higher initial cost of purchase. This study is aimed at estimating the clinical and economic consequences of using CSII as compared to DMI by means of a cost-utility analysis.

Methods: A mathematical simulation model was adapted using nationwide clinical and economic data to simulate the long-term clinical and economic consequences for a DM1 patient. The time horizon was the patient's lifetime, including only direct healthcare costs and updating both costs and benefits at an annual $3 \%$ rate.

Results: In the basecase, the patients treated using CSII gained 0.890 years $(\mathrm{p}<0.05)$ and 0.852 QALYs $(\mathrm{p}<0.05)$. CSII treatment gives rise to an incremental average cost of $25,523 €(\mathrm{p}<0.05)$ per patient treated, which gave us an incremental cost- utility ratio of $29,947$ €lQALY [CI 95\% $(29,519 ; 30,375)]$.

Conclusions: The improvement in the glucose control among those patients treated using CSII was related to an overall lower cost in the handling of DM1 patients, which was found to have a favourable cost-utility ratio in comparison to conventional MDI treatment.

Key words: Diabetes Mellitus Type 1. Cost-utility. Insulin pump. Programmable implantable. Economic model. 


\section{INTRODUCCIÓN}

La diabetes mellitus (DM) es una de las patologías crónicas que tienen un gran impacto tanto a nivel social como económico, suponiendo una importante carga para el sistema sanitario ${ }^{1}$. Este hecho no se debe exclusivamente a su alta prevalencia sino que es consecuencia del riesgo asociado a desarrollar complicaciones de carácter grave o incluso la muerte. De hecho en España la DM ha llegado a representar la tercera causa de muerte entre la población femenina ${ }^{2}$.

Los pacientes con diabetes mellitus tipo 1 (DM1) han podido mejorar su control mediante terapias intensivas de insulina, utilizando múltiples dosis (MDI) o mediante bombas de infusión continua de insulina (BICI). En España la incidencia de la DM1 se ha estimado en torno a 11 ó 12 casos por cada 100.000 personas año ${ }^{3}$.

Introducidas hace 30 años como una alternativa terapéutica a las inyecciones de insulina, las BICI han sufrido una evolución que ha conseguido que actualmente sea posible disponer de dispositivos más pequeños y seguros.

Varios estudios publicados recientemente han demostrado que el uso de BICI está asociado a un mejor control glucémico, incluyendo un menor número de eventos hipoglucémicos comparándolo con las $\mathrm{MDI}^{4-7}$. Sin embargo todavía es limitado debido principalmente al requerimiento de una inversión inicial mayor frente a las MDI.

En el entorno actual, en el que se llevan a cabo importantes esfuerzos por controlar el gasto sanitario, la inversión en este tipo de terapias debería estar justificada tanto clínica como económicamente. Hasta la actualidad no se ha publicado en España ninguna evaluación económica de la eficiencia del uso de las BICI frente a las MDI.

El presente estudio pretende cubrir esta laguna, adaptando un modelo de simulación para pacientes diabéticos, con el objeto de estudiar las consecuencias clínicas y económicas a largo plazo del uso de BICI frente a MDI en pacientes con DM1 en España, mediante un análisis de coste-utilidad.

\section{MATERIAL Y MÉTODOS}

\section{EI modelo de CORE}

Para poder estimar los costes y los beneficios de las terapias comparadas a largo plazo se proyectaron los resultados a través de un modelo matemático. Los modelos son herramientas flexibles que de forma simplificada representan una realidad experimentada con base a una teoría. Utilizar modelos de patologías en el ámbito sanitario ha sido ampliamente discutido y se percibe como una herramienta útil para ayudar a la toma de decisiones en situaciones de incertidumbre ${ }^{8-10}$.

Para llevar a cabo nuestro objetivo se adaptó para el entorno español el modelo de diabetes de CORE. El modelo de CORE ha sido validado y ampliamente usado a nivel internacional para la evaluación de diferentes estrategias de tratamientos en pacientes diabéticos. Por problemas de limitación de espacio no es posible describir en profundidad los detalles del modelo, por lo que nos remitimos a dos publicaciones recientes en las que se detallan tanto sus características como la validación que se llevó a cabo para estudiar su ajuste ${ }^{11,12}$.

A modo de resumen, el modelo simula los principales acontecimientos que puede sufrir un paciente con DM1 a través de diferentes submodelos de Markov, que toman datos clínicos y económicos de diferentes estudios publicados y que relacionados entre si simulan la evolución clínica de un paciente con DM1, teniendo en cuenta tanto el perfil de riesgo de la población que se va a estudiar, como la estrategia de tratamiento que sigue cada uno de los pacientes. En la tabla 1 se presentan los diferentes submodelos que se incluyen en el análisis, así como los dife- 
Tabla 1

Descripción de los diferentes submodelos que simulan las diferentes patologías asociadas a un paciente con DM1

\begin{tabular}{|c|c|c|}
\hline Submodelos & Supuestos probabilidades de transición & Ref \\
\hline $\begin{array}{l}\text { Infarto de miocardio (IM): No IM; IM; Muerte } \\
\text { por IM }\end{array}$ & $\begin{array}{l}\text { Ajuste con la ecuación de Framingham teniendo en cuenta otras variables clínicas } \\
\text { como el sexo, la historia de enfermedades cardiovasculares, condición de fumador o } \\
\text { hipertensión. }\end{array}$ & $(13)$ \\
\hline Angina: No angina; Historia de angina & $\begin{array}{l}\text { Ajuste con la ecuación de Framingham teniendo en cuenta otras variables clínicas } \\
\text { como el sexo, la historia de enfermedades cardiovasculares, condición de fumador o } \\
\text { hipertensión. }\end{array}$ & $(13)$ \\
\hline $\begin{array}{l}\text { Insuficiencia cardiaca (IC): No IC; IC; Muerte } \\
\text { por IC }\end{array}$ & $\begin{array}{l}\text { Ajuste con la ecuación de Framingham teniendo en cuenta otras variables clínicas } \\
\text { como el sexo, la historia de enfermedades cardiovasculares, condición de fumador o } \\
\text { hipertensión. }\end{array}$ & $(13)$ \\
\hline $\begin{array}{l}\text { Infarto cerebral: No infarto; Infarto; Muerte por } \\
\text { infarto }\end{array}$ & $\begin{array}{l}\text { Ajuste con la ecuación de Framingham teniendo en cuenta otras variables clínicas } \\
\text { como el sexo, la historia de enfermedades cardiovasculares, condición de fumador o } \\
\text { hipertensión. }\end{array}$ & (13) \\
\hline $\begin{array}{l}\text { Transtorno vascular periérico (TPV): No TVP; } \\
\text { TVP }\end{array}$ & $\begin{array}{l}\text { Ajuste con la ecuación de Framingham teniendo en cuenta otras variables clínicas } \\
\text { como el sexo, la historia de enfermedades cardiovasculares, condición de fumador o } \\
\text { hipertensión. }\end{array}$ & (13) \\
\hline Neuropatía: No neuropatía; Neuropatía & $\begin{array}{l}\text { Probabilidades de transición en el estudio DCCT, ajustadas por variables clínicas de } \\
\text { los individuos como edad, sexo o tiempo de diabetes }\end{array}$ & $\begin{array}{l}(14- \\
16)\end{array}$ \\
\hline $\begin{array}{l}\text { Retinopatía: No retinopatía; Antecedentes de } \\
\text { retinopatía; Retinopatía proliferativa; Pérdida de } \\
\text { visión severa }\end{array}$ & $\begin{array}{l}\text { Probabilidades de transición en el estudio DCCT, ajustadas por variables clínicas de } \\
\text { los individuos como edad, sexo o tiempo de diabetes }\end{array}$ & $\begin{array}{l}(14- \\
16)\end{array}$ \\
\hline $\begin{array}{l}\text { Edema macular }(\boldsymbol{E M}) \text { : No EM; EM; Pérdida de } \\
\text { visión severa }\end{array}$ & $\begin{array}{l}\text { Probabilidades de transición en el estudio DCCT, ajustadas por variables clínicas de } \\
\text { los individuos como edad, sexo o tiempo de diabetes }\end{array}$ & $\begin{array}{l}(14- \\
16)\end{array}$ \\
\hline $\begin{array}{l}\text { Nefropatía: Sin complicación renal; } \\
\text { Microalbuminuria; Macroalbuminuria; } \\
\text { Enfermedad renal Terminal; Diálisis; Transplante } \\
\text { renal; Muerte }\end{array}$ & $\begin{array}{l}\text { Probabilidades de transición en el estudio DCCT, ajustadas por variables clínicas de } \\
\text { los individuos como edad, sexo o tiempo de diabetes }\end{array}$ & $\begin{array}{l}(14- \\
16)\end{array}$ \\
\hline Hipoglucemia: Sobrevive; Muere & $\begin{array}{l}\text { Probabilidades de transición en el estudio DCCT, ajustadas por variables clínicas de } \\
\text { los individuos como edad, sexo o tiempo de diabetes }\end{array}$ & $\begin{array}{l}(14- \\
16)\end{array}$ \\
\hline Cetoacidosis: Sobrevive; Muere & $\begin{array}{l}\text { Probabilidades de transición en el estudio DCCT, ajustadas por variables clínicas de } \\
\text { los individuos como edad, sexo o tiempo de diabetes }\end{array}$ & $\begin{array}{l}(14- \\
16)\end{array}$ \\
\hline Ácido láctico: Sobrevive; Muere & $\begin{array}{l}\text { Probabilidades de transición en el estudio DCCT, ajustadas por variables clínicas de } \\
\text { los individuos como edad, sexo o tiempo de diabetes }\end{array}$ & $\begin{array}{l}(14- \\
16)\end{array}$ \\
\hline $\begin{array}{l}\text { Pie diabético: No úlcera; Úlcera no infectada; } \\
\text { Úlcera infectada; Úlcera curada; Úlcera } \\
\text { recurrente no infectada; Úlcera recurrente } \\
\text { infectada; Gangrena; Historia de amputación; } \\
\text { Muerte }\end{array}$ & $\begin{array}{l}\text { Probabilidades de transición relacionadas con el trastorno vascular periférico y la } \\
\text { nefropàtía }\end{array}$ & $(15)$ \\
\hline $\begin{array}{l}\text { Cataratas: No cataratas; Primera catarata con } \\
\text { operación; Segunda catarata con operación }\end{array}$ & Probabilidades de transición basadas en un estudio publicado para población inglesa & $(17)$ \\
\hline
\end{tabular}

rentes estados de salud que contemplan cada uno de ellos. Las probabilidades de transición están basadas en diferentes estudios clínicos ${ }^{13-17}$ y varían con el tiempo y con el perfil de riesgo de la población de estudio.

Dado que el análisis que se pensó adoptar fue el de coste-utilidad, a cada uno de los estados de salud contemplados en los dife- rentes submodelos se le asignó un valor de utilidad. Para algunos de los estados de salud no fue posible por lo que se optó por tomar como valor por defecto el de un paciente diabético sin complicaciones. En la tabla 2 se presentan todos los estados de salud contemplados en los diferentes submodelos de Markov, así como el valor de la utilidad asociada a cada uno de esos estados. 
Tabla 2

Valores de utilidades utilizados en cada uno de los estados de salud que contemplan los diferentes submodelos

\begin{tabular}{|c|c|c|}
\hline Descripción del estado & Utilidad/Desutilidad & Ref \\
\hline Diabetes sin complicaciones & 0,814 & $(42)$ \\
\hline Infarto de Miocardio, primer año del evento & $-0,129$ & $(42)$ \\
\hline Infarto de Miocardio, años siguientes & 0,736 & (42) \\
\hline Angina & 0,682 & $(42)$ \\
\hline Insuficiencia cardiaca & 0,633 & $(42)$ \\
\hline Ictus, primer año del evento & $-0,181$ & $(42)$ \\
\hline Ictus, años siguientes & 0,545 & $(42)$ \\
\hline Trastorno vascular periférico & 0,570 & (43) \\
\hline Microalbuminuria & 0,814 & a \\
\hline Macroproteinuria & 0,814 & a \\
\hline Hemodialisis & 0,490 & (44) \\
\hline Diálisis peritoneal & 0,560 & (44) \\
\hline Transplante renal & 0,762 & (44) \\
\hline Retinopatía diabética inicial & 0,814 & a \\
\hline Retinopatía diabética proliférica & 0,794 & (43) \\
\hline Edema macular & 0,794 & $(43)$ \\
\hline Pérdida de visión severa / ceguera & 0,734 & (44) \\
\hline Catarata & 0,794 & (43) \\
\hline Neuropatía & 0,624 & (43) \\
\hline Úlcera diabética curada & 0,814 & $\mathrm{a}$ \\
\hline Úlcera activa & 0,600 & $(45)$ \\
\hline Amputación extremidad inferior, primer año del evento & $-0,109$ & $(42)$ \\
\hline Amputación extremidad inferior, años siguientes & 0,680 & $(42)$ \\
\hline Evento hipoglucémico & $-0,0052$ & $(46)$ \\
\hline
\end{tabular}

(a) No se identificó un valor de utilidad concreto por lo que se asumió el mismo valor que el estado de salud de Diabetes sin complicaciones. 


\section{Las terapias comparadas}

El Diabetes Control and Complications Trial (DCCT) ${ }^{18}$ demostró que la terapia intensiva, bien por inyecciones múltiples o bien por infusión subcutánea con bomba externa, retrasaba la aparición y demoraba el progreso de las complicaciones diabéticas en comparación con la terapia convencional.

El estudio $\mathrm{EDIC}^{19}$, que representa 8 años adicionales de seguimiento del DCCT, demostró que a lo largo del tiempo los niveles glucémicos de los pacientes en terapia convencional comenzaron a disminuir mientras que los del grupo que recibió terapia intensiva aumentaron. En general, los niveles glucémicos de ambos grupos se fueron igualando a los 8 años del estudio. No obstante, a pesar de esa similitud en los valores glucémicos, los pacientes que formaron el grupo de terapia intensiva presentan a largo plazo mayores beneficios en términos de protección renal. Por ello, la conclusión fue que los beneficios de una terapia intensiva frenan la progresión de la enfermedad renal muchos años después de que el intensivo control glucémico haya finalizado.

Tanto el DCCT como el EDIC no fueron estudios diseñados para comparar las diferencias en los resultados proporcionados por el uso de BICI frente a DMI, sino para comparar la terapia convencional frente a la terapia intensiva, por ello para el presente estudio la mejor evidencia disponible fue un metanálisis que comparaba directamente el uso de BICI con $\mathrm{DMI}^{7}$ publicado en 2003, y que recoge 52 estudios con un total de más de 1.500 pacientes diagnosticados de DM1.

En dicho meta-análisis se concluye que el uso de BICI frente a DMI mejora el control de la $\mathrm{HbA}_{1 \mathrm{c}}$ en un $1,2 \%$, aunque también se asocia con una ganancia de peso, reflejada en un aumento del índice de masa corporal (IMC) de $1,03 \mathrm{~kg} / \mathrm{m}^{-2}$.

Con respecto al riesgo de sufrir eventos hipoglucémicos así como la tasa de eventos de cetoacidosis este meta-análisis concluye que el uso de BICI no parece estar asociado a un mayor riesgo de sufrir ninguno de estos eventos ${ }^{7}$.

Dicho meta-análisis ${ }^{7}$ presenta diferentes limitaciones, tal y como argumentan sus autores. Por un lado, la mayor limitación es que gran parte de los estudios que se contemplan en el mismo fueron llevados a cabo antes de 1987 y por lo tanto utilizan tecnología que ha quedado obsoleta. Por otro lado, por problemas de la heterogeneidad de la información disponible, en algunos aspectos no es posible alcanzar conclusiones robustas, como es el caso del riesgo de eventos hipoglucémicos o del riesgo relacionado con el número de eventos de cetoacidosis.

\section{Población de estudio}

Se llevó a cabo una simulación con una cohorte hipotética de 1.000 pacientes con cada una de las dos terapias contempladas en el modelo. A esta cohorte se le asignó unas características demográficas basales, factores de riesgo y complicaciones previas, obtenidas a partir de datos observacionales del registro de pacientes en tratamiento con BICI que se lleva a cabo en Cataluña desde 1998-2004 ${ }^{20}$ (tabla 3).

\section{Perspectiva del estudio, datos clínicos y económicos}

La perspectiva del estudio fue la del proveedor de servicios sanitarios, esto es, el Sistema Nacional de Salud (SNS), por lo que sólo se tuvieron en cuenta los costes directos sanitarios. Dado que la edad media de los pacientes de la simulación fue de 35,9 años (tabla 3), se tuvo en cuenta como horizonte temporal el de toda la vida del paciente. Para ello el modelo simula los diferentes acontecimientos que puede sufrir un paciente con DM1 con un perfil clínico y sociodemográfico definido, y acaba la simulación cuando el 
Tabla 3

Características demográficas y clínicas de la cohorte de pacientes simulada en el caso base. Datos obtenidos a partir registro de pacientes en tratamiento con BICI en Cataluña desde 1998-2004

\begin{tabular}{|l|l|}
\hline Características demográficas y clínicas & Valor \\
\hline Género & \\
Hombre & $30 \%$ \\
Mujer & $70 \%$ \\
\hline Edad media $( \pm$ d.s.) & $35,9 \pm 12,3$ \\
\hline Años desde el diagnóstico & 15,2 \\
\hline HbAlc \pm d.e.) & $8,3 \pm 1,4$ \\
\hline Hospitalizaciones durante el último año & \\
Ninguna & $67,7 \%$ \\
Una & $22,8 \%$ \\
Dos & $9,5 \%$ \\
\hline Complicaciones & \\
Hipoglucemias graves & $42,4 \%$ \\
Cetoacidosis & $8,3 \%$ \\
Retinopatía & $31,9 \%$ \\
Ceguera & $0,7 \%$ \\
Fotocoagulación & $22,6 \%$ \\
Hemorragia vítrea & $4,2 \%$ \\
Vitrectomía & $3,2 \%$ \\
Nefropatía & $14,6 \%$ \\
Transplantes o hemodiálisis & $1,5 \%$ \\
Neuropatía & $19,4 \%$ \\
Vasculopatia periférica & $2,2 \%$ \\
Amputaciones & $0,3 \%$ \\
Cardiopatía isquemica & $2,8 \%$ \\
Accidente vascular cerebral & $1,2 \%$ \\
\hline Complicaciones con BICI & \\
\hline Obstrucción & $3,0 \%$ \\
\hline
\end{tabular}


Tabla 4

Relación de costes incluidos en el modelo, que contemplan la totalidad de las complicaciones que puede experimentar un paciente con DM1. Los costes están expresados en euros del 2005, y corresponden a costes por evento, o en su caso, costes anuales de tratamiento

\begin{tabular}{|c|c|c|}
\hline Descripción del evento o estado & $\begin{array}{c}\text { Coste } \\
(€, 2005)\end{array}$ & Ref \\
\hline Infarto de Miocardio, primer año del evento & 19.276 & $(47)$ \\
\hline Infarto de Miocardio, años siguientes & 776 & $(47)$ \\
\hline Angina, primer año del evento & 2.207 & $(48)$ \\
\hline Angina, años siguientes & 923 & $(49)$ \\
\hline Insuficiencia cardiaca, primer año del evento & 3.737 & $(50)$ \\
\hline Insuficiencia cardiaca, años siguientes & 709 & $(50)$ \\
\hline Ictus mortal & 3.201 & $(51)$ \\
\hline Ictus, primer año del evento & 4.638 & $(51)$ \\
\hline Ictus, años siguientes & 1.722 & $(51)$ \\
\hline Enfermedad vascular periférica, por evento & 2.008 & $(52)$ \\
\hline Hemodiálisis & 31.233 & $(51)$ \\
\hline Diálisis perioteneal & 32.706 & $(51)$ \\
\hline Transplante renal, primer año & 28.370 & $(53)$ \\
\hline Transplante renal, años siguientes & 8.337 & $(53)$ \\
\hline Tratamiento con láser & 176 & $(54)$ \\
\hline Extracción de cataratas & 1.041 & $(48)$ \\
\hline Seguimiento anual cataratas & 915 & $(55)$ \\
\hline Neuropatía, primer año del evento & 2.531 & $(48)$ \\
\hline Úlcera sin infección & 1.142 & $(56)$ \\
\hline Úlcera con infección & 1.999 & $(56)$ \\
\hline Gangrena & 5.611 & $(48)$ \\
\hline Amputación extremidad inferior, primer año del evento & 14.786 & $(57)$ \\
\hline Evento hipoglucémico severo & 338 & $(57)$ \\
\hline Cetoacidosis & 997 & $(58)$ \\
\hline Coste anual tratamiento BICI & 3.773 & (a) \\
\hline Coste anual tratamiento MDI & 2.087 & (b) \\
\hline
\end{tabular}

(a) Coste total anual de tratamiento, que incluye el coste del dispositivo según catálogo (facilitado por Medtronic Ibérica S.A.) más el coste de los materiales desechables, así como el consumo de insulina Al dispositivo se asignó una vida útil de ocho años, por lo que el cose imputado incluye la amortización mensual de dicho coste.

(b) Coste total anual de tratamiento, que incluye el coste de los materiales desechables, así como el consumo de insulina 
paciente fallece. De esta forma los pacientes simulados tienen la posibilidad de desarrollar todas las complicaciones contempladas en el modelo y se puede estimar la esperanza de vida de cada una de las dos cohortes estudiadas (BICI y MDI).

Tanto los costes como beneficios fueron calculados a una tasa del $3 \%$ anual en el caso base y actualizados a euros del $2005^{21}$.

En la tabla 4 se muestran los datos de costes utilizados para cada una de los posibles eventos que puede sufrir un paciente con DM1 contemplados en el modelo. Estos costes han sido extraídos a partir de una revisión de estudios publicados para el entorno español. De forma individualizada para cada uno de los valores de los costes de la tabla 4 se identifica la referencia bibliográfica. Los costes anuales de las terapias evaluadas corresponden a costes anuales de tratamiento con cada una de ellas. Los datos de consumo de insulina así como de material desechable por parte de los pacientes con DMI se obtuvieron directamente analizando el consumo que tenían los pacientes seleccionados del registro antes de la implantación de una $\mathrm{BICI}^{20}$.

El coste anual de tratamiento con BICI que se muestra en la tabla 4 incluye el coste de los materiales desechables, consumo de insulina y el coste del dispositivo según catálogo (facilitado por Medtronic Ibérica S.A.). Al dispositivo se asignó una vida útil de ocho años, por lo que el coste imputado incluye su amortización mensual.

Con el fin de poder recoger la incertidumbre de los parámetros incluidos en el modelo se utilizó un método de simulación no paramétrico conocido como muestreo reiterado (bootstrapping) ${ }^{22}$. Para el caso base asumimos que la tasa de reducción de eventos hipoglucémicos y la tasa de cetoacidosis fueron las mismas para ambos tratamientos y estuvieron basadas en los resultados del $\mathrm{DCCT}^{23}$. Sin embargo resultados prelimina- res del estudio 5 Naciones ${ }^{24}$ han demostrado que en los resultados correspondientes al ámbito español el uso de BICI redujo en un $66 \%$ la incidencia de eventos hipoglucémicos. Los resultados de este estudio están en la misma línea que otros publicados recientemente ${ }^{7,25-28}$, los cuales apuntan a que el uso de BICI respecto a DMI reduce la tasa de eventos hipoglucémicos así como el riesgo de cetoacidosis.

Para estudiar la sensibilidad de los resultados del modelo ante variaciones en sus principales parámetros, se llevaron a cabo diferentes análisis de sensibilidad univariantes, variando los valores de algunas de las variables clave del modelo. Las variables elegidas para el análisis de sensibilidad fueron: tasa de reducción del riesgo hipoglucémico del $66 \%$ en pacientes con $\mathrm{BICI}^{24}$. Dado que existen ciertas discrepancias ante la necesidad de descontar tanto costes como beneficios, a iguales o diferentes tasas de descuento $^{29}$, en el análisis de sensibilidad se dan resultados con una tasa del 5\% tanto para costes como beneficios y alternativamente asumiendo un $3 \%$ para costes y un $0 \%$ para resultados. Como se ha comentado, en el metanálisis en el que se ha basado nuestro estudio se concluyó que el uso de BICI inducía un aumento en el peso de los pacientes, medido a través del índice de masa corporal (IMC), por lo que para el análisis de sensibilidad se tuvo en cuenta su efecto en los resultados asumiendo que los pacientes no tenían esta ganancia de peso. Otros metanálisis han estimado que el uso de BICI frente a MDI producía reducciones de la $\mathrm{HbA1c}$ menores a las encontradas en el metanálisis que se usó de base ${ }^{7}$, de esta forma para el análisis de sensibilidad se tuvo en cuenta una reducción del $0,51 \%$ en el control glucémico tal y como se reportó en el estudio de Pickup ${ }^{26}$.

Todos los resultados se presentan como valores medios \pm intervalo de confianza (IC) al 95\%. Para detectar diferencias estadísticamente significativas en los valores medios 
para las variables en cada grupo de estudio se llevó a cabo la prueba t para igualdad de medias asumiendo varianzas diferentes.

\section{RESULTADOS}

En el caso base el modelo proyectó una esperanza de vida superior en la cohorte de pacientes tratados con BICI, 16,827 $\pm 0,012$ años frente a $15,937 \pm 0,012$ años para los pacientes tratados con DMI ( $\mathrm{p}<0,005)$. En el caso de los años de vida ajustados por calidad (AVAC) el modelo también proyectó una diferencia de 0,852 $\pm 0,011(\mathrm{p}<0,005)$ AVACs en los pacientes tratados con BICI frente a DMI.

Respecto a las complicaciones contempladas en el modelo, en las figuras 1-4 se han representado los resultados referentes a la tasa de incidencia acumulada de algunas de las complicaciones que se contemplan en el modelo. Sin entrar mucho en detalle, en complicaciones que se sabe que están muy relacionadas con el control glucémico, como es el caso de las complicaciones renales, oculares, o la relacionada con el pie diabético, en la cohorte de pacientes con BICI se tiene una menor incidencia de este tipo de eventos (figuras 1-3).
En el caso de las complicaciones cardiovasculares (figura 4), se aprecia que hay una mayor tasa de incidencia en pacientes tratados con BICI. Una posible explicación de este hecho es que el uso de BICI aumenta la esperanza de vida en esta cohorte de pacientes, y la mejora en el control glucémico que proporcionan las BICI no beneficia en la misma magnitud la prevención de eventos cardiovasculares, dado que a lo largo del tiempo nos encontramos que en la cohorte de BICI hay un mayor número de pacientes debido a que la esperanza de vida es más alta, el número de eventos cardiovasculares que se presentarán en esta cohorte será también mayor.

En la tabla 5 se presentan los resultados del análisis coste-utilidad para el caso base. El coste medio por paciente tratado en la cohorte de BICI fue de $105.439 \pm 99,1 €$ frente a los 79.916 $\pm 96,8 €$ en la cohorte de pacientes tratado con DMI ( $\mathrm{p}<0,005)$. Con estos resultados, el ratio coste - utilidad incremental (RCUI) estimado fue de 29.947 $\pm 428 €$ por AVAC.

En la figura 5 se presentan los resultados del plano coste-efectividad. Esta figura muestra los diferentes resultados que se han obtenido a lo largo de las 1.000 simulaciones

Figura 1

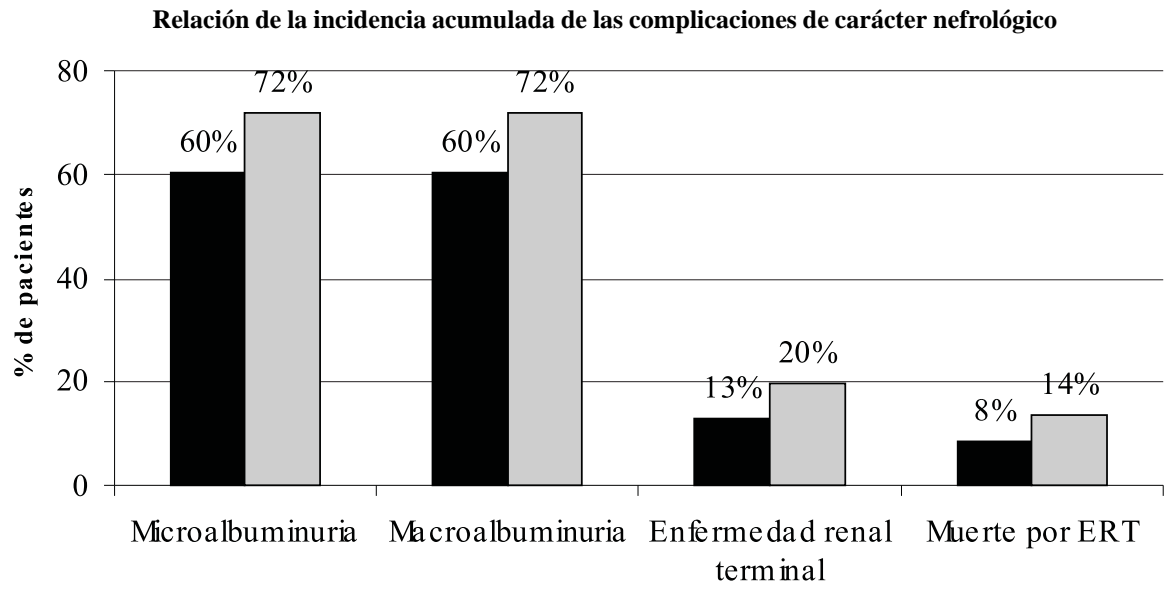

Rev Esp Salud Pública 2006, Vol. 80, N. ${ }^{\circ} 6$ 
Figura 2

Relación de la incidencia acumulada, relaciones de las complicaciones oculares

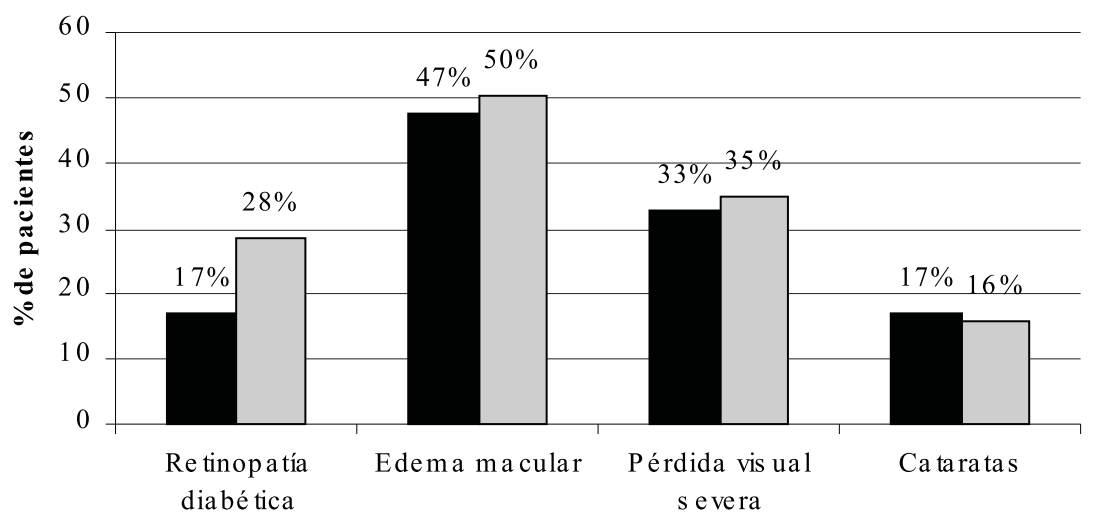

Figura 3

Relación de la incidencia acumulada relacionada con el pie diabético

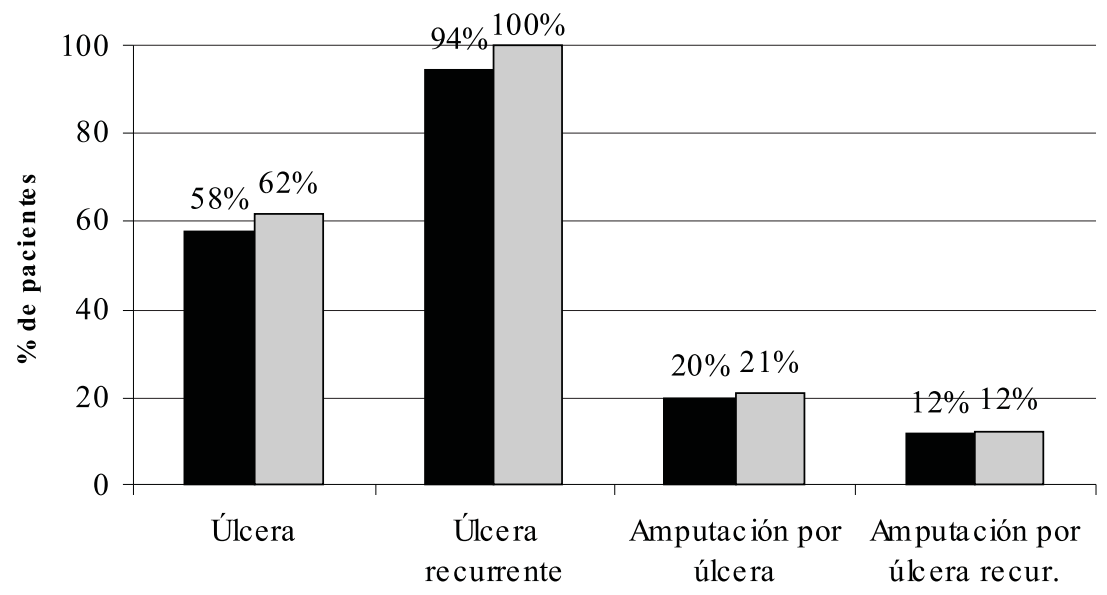

que se llevaron a cabo para el caso base, y como puede apreciarse, gran parte de las simulaciones se sitúan por debajo de 30.000 $€$ AVAC.

Como se ha comentado anteriormente, a la hora de desarrollar el presente estudio se contó con ciertas limitaciones e incertidumbre respecto de la información clínica disponible. Para tratar de mitigar la importancia potencial de esta situación se realizó un aná- lisis de sensibilidad en el que se han identificado las variables que a priori y de acuerdo con la literatura podrían tener más influencia en los resultados este estudio y se realizó con ellas un análisis de sensibilidad univariante. En la misma tabla 5 se muestran también los resultados de estos análisis adicionales.

Así pues, cuando aplicamos una tasa de descuento mayor, tanto a costes como a resultados clínicos el RCUI se incrementó 
Tabla 5

Resumen de los resultados del análisis coste-efectividad para el caso base y para los diferentes análisis de sensibilidad llevados a cabo

\begin{tabular}{|c|c|c|c|}
\hline & Coste medio $(€)$ & AVAC & RCUI (€/ AVAC) \\
\hline \multicolumn{4}{|c|}{ Caso base } \\
\hline BICI & $105.439 \pm 99,1$ & $11,135 \pm 0,008$ & \\
\hline DMI & $79.916 \pm 96,8(\mathrm{p}<0,005)$ & $10,283 \pm 0,008(\mathrm{p}<0,005)$ & $29.947 \pm 428$ \\
\hline \multicolumn{4}{|c|}{ Tasa de descuento } \\
\hline \multicolumn{4}{|c|}{$5 \%$ costes y $5 \%$ beneficios } \\
\hline $\mathrm{BICI}$ & $79.659 \pm 68,9$ & $8,877 \pm 0,01$ & \\
\hline DMI & $59.953 \pm 68,9(\mathrm{p}<0,005)$ & $8,289 \pm 0,01(\mathrm{p}<0,005)$ & $33.497 \pm 531,4$ \\
\hline \multicolumn{4}{|c|}{$3 \%$ costes y $0 \%$ beneficios } \\
\hline BICI & $105.440 \pm 99,1$ & $16,923 \pm 0,02$ & \\
\hline DMI & $79.916 \pm 96,9(\mathrm{p}<0,005)$ & $15,256 \pm 0,01(\mathrm{p}<0,005)$ & $15.317 \pm 210$ \\
\hline \multicolumn{4}{|c|}{ Efecto de las BICI en la $\mathrm{HbA}_{1 \mathrm{c}}-0,51 \%$ puntos } \\
\hline \multicolumn{4}{|c|}{$106647+1042$} \\
\hline DMI & $79.916 \pm 96,8(\mathrm{p}<0,005)$ & $10,28 \pm 0,007(\mathfrak{p}<0,005)$ & $103.584 \pm 132.656$ \\
\hline \multicolumn{4}{|c|}{ No ganancia de peso } \\
\hline BICI & $105.524 \pm 100,9$ & $11,14 \pm 0,01$ & \\
\hline DMI & $79.916 \pm 96,9(\mathrm{p}<0,005)$ & $10,28 \pm 0,01(\mathrm{p}<0,005)$ & $29.718 \pm 387,3$ \\
\hline \multicolumn{4}{|c|}{ Reducción eventos hipoglucémicos $66 \%$} \\
\hline \multicolumn{4}{|c|}{$102542+1022$} \\
\hline DMI & $79.916 \pm 96,8(\mathrm{p}<0,005)$ & $10,28 \pm 0,01(\mathrm{p}<0,005)$ & $25.680 \pm 356,8$ \\
\hline
\end{tabular}

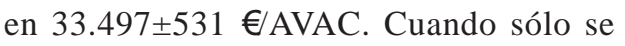
aplicó una tasa de descuento del $3 \%$ en los costes el RCUI experimentó una disminución considerable, pasando a ser de $15.317 \pm$ $210 €$ AVAC.

La variable que se detectó que más influye en los resultados del modelo es la tasa de mejora de la HbA1c. De acuerdo con los resultados de Pickup ${ }^{26}$ se asumió un beneficio de $-0,51 \%$ sobre el cambio en los niveles de la HbA1c para los pacientes con BICI, y se obtuvo un RCUI de 103.584 $\$ 32.656$ $€$ AVAC. Cuando no se tuvo en cuenta el hecho de que los pacientes con BICI experimentan una ligera ganancia de peso, los resultados del RCUI fueron de $29.718 \pm 387$ $€$ AVAC, muy similares a los obtenidos en el escenario base. La reducción de los eventos hipoglucémicos tuvo un efecto significativo en los resultados del modelo, cuando se supuso una reducción del $66 \%$ de este tipo de eventos en los pacientes con BICI, el RCUI se redujo a $25.680 \pm 357 €$ AVAC.

\section{DISCUSIÓN}

El objetivo del presente estudio fue evaluar clínica y económicamente el uso de BICI frente a DMI a largo plazo, en pacientes con DM1. Para poder proyectar los resultados a largo plazo se ha usado un modelo matemático que simula los principales acontecimientos que puede sufrir un paciente diabético ${ }^{11}$. El uso de modelos también permite 
Figura 4

Relación de la incidencia acumulada relacionada con complicaciones cardiovasculares

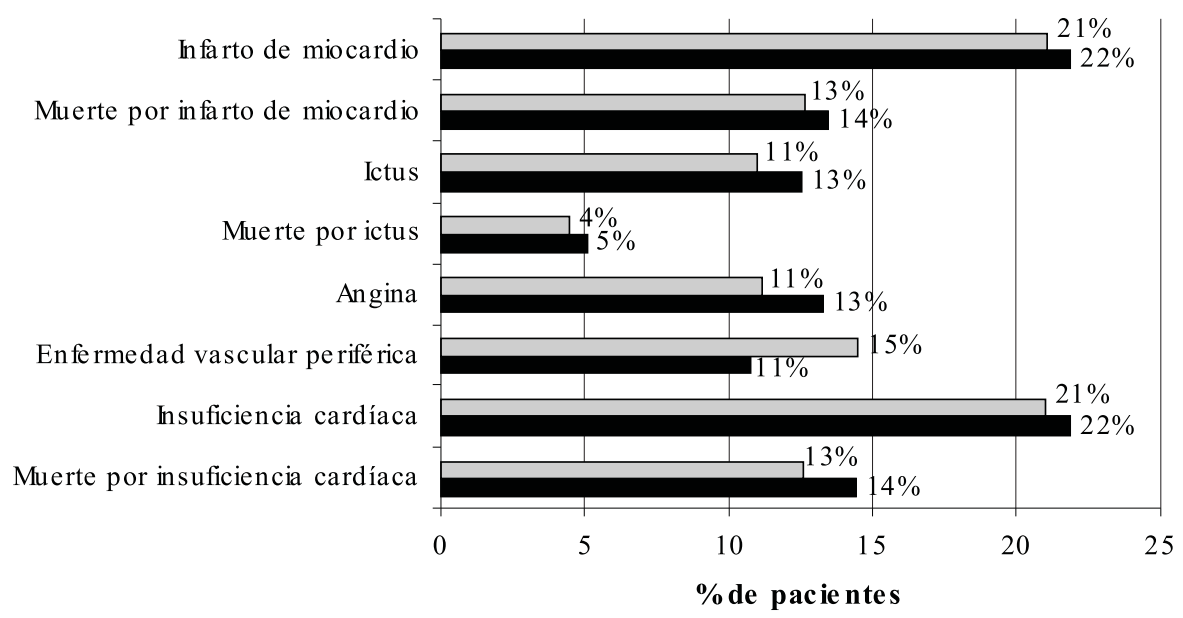

Figura 5

Plano coste-efectividad incremental que expone los resultados de todas las simulaciones de Montecarlo llevadas a cabo

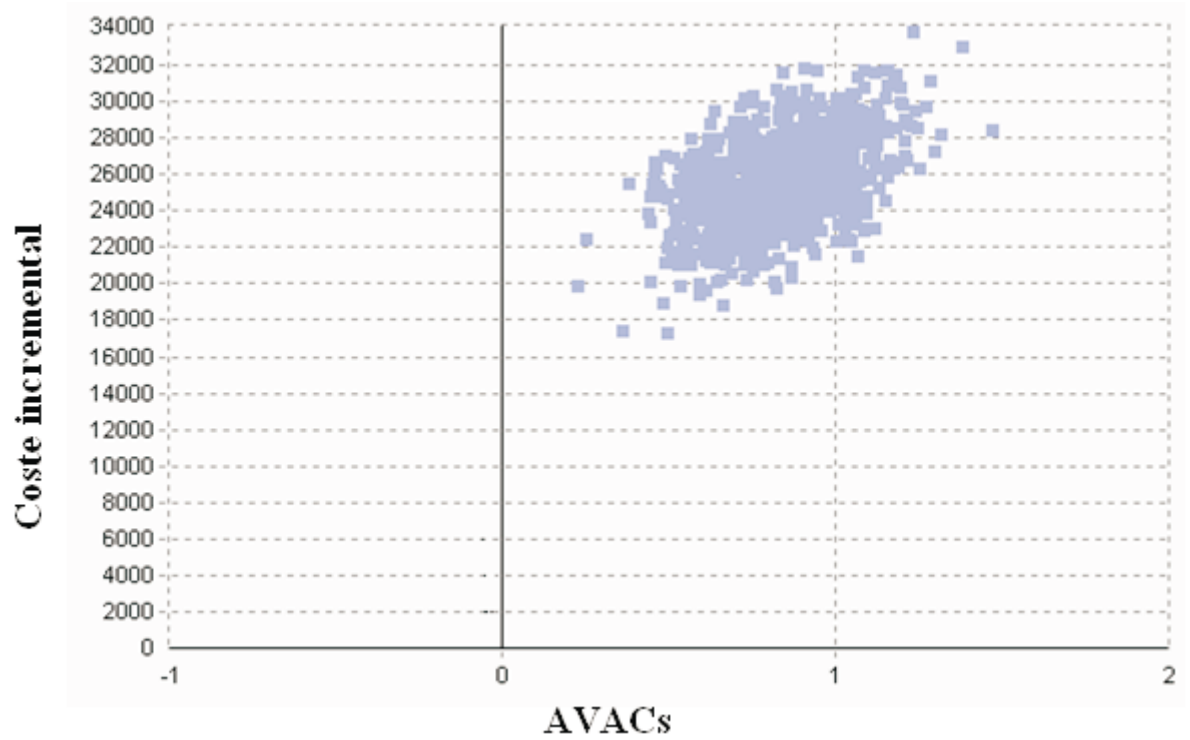

estudiar los efectos de las terapias comparadas más allá del horizonte temporal considerado en el ensayo. De otro modo sería nece- sario llevar a cabo ensayos con un seguimiento de muchos años, con el coste y las limitaciones que ello supone. Para el presen- 
te estudio las principales variables clínicas necesarias para la elaboración del modelo han sido básicamente extraídas del metanálisis llevado a cabo por Weissberg-Benchell y col. ${ }^{7}$, en el que se comparó el tratamiento con BICI frente a DMI en pacientes con DM1. El seguimiento medio del uso de BICI en los estudios incluidos en dicho metanálisis fue alrededor de un año, y la principal conclusión fue que el uso de BICI produjo una mejora en el control glucémico de los pacientes respecto a DMI. Este resultado fue usado en nuestro modelo de simulación y dado que el horizonte temporal fue toda la vida del paciente se tuvo que suponer que dicha diferencia en el control glucémico se mantenía a lo largo de toda la vida del paciente, a pesar de que no se dispone de una evidencia robusta al respecto. Esta es una de las limitaciones del presente estudio, sin embargo diversos estudios apuntan a que la mejora en el control glucémico que aporta el uso de BICI con respecto a DMI se mantiene a lo largo del tiempo. Por ejemplo en el estudio de Bruttomesso y col. ${ }^{30}$ se estudió un grupo de 138 pacientes en la región italiana de Véneto y se demostró que la tasa de reducción en el $\mathrm{HbA}_{1 \mathrm{c}}$ registrada durante el primer año de tratamiento con BICI $(-1,4 \%)$ fue mantenida durante un periodo de 10 años, reduciéndose el número de eventos hipoglucémicos severos, cetoacidosis, consultas y admisiones hospitalarias.

Para nuestro análisis tuvimos en cuenta el metanálisis de Weissberg-Benchell y col. ${ }^{7}$ que a pesar de presentar algunas limitaciones que ya se han comentado a lo largo de este artículo, provee el mejor grado de evidencia disponible. Una de las limitaciones de este metanálisis ${ }^{7}$ es que la heterogeneidad en los resultados de los diferentes estudios hizo que no se pudieran alcanzar conclusiones robustas para algunas variables, como fue la reducción de los eventos hipoglucémicos, la ganancia de peso debida al uso de BICI, o la tasa de eventos de cetoacidosis. Para poder contemplar estos aspectos, se llevó a cabo una serie de análisis de sensibili- dad con dichas variables con el objeto de estudiar su influencia en los resultados del modelo.

En el análisis de sensibilidad se detectó que la variable que más incertidumbre introduce en el modelo fue la relativa a la mejora del control de la $\mathrm{HbA}_{1 \mathrm{c}}$ con el tratamiento con BICI. En el escenario base se asumió una tasa del $-1,2 \%$ de acuerdo a los resultados obtenidos en el metanálisis que se usó de referencia ${ }^{7}$, sin embargo cuando se supuso un valor de $-0,51 \%$ para el análisis de sensibilidad $^{26}$ los resultados obtenidos para el RCUI fueron de 103.584 $\pm 132.656 €$ IAVAC, obteniéndose una gran variabilidad tal y como refleja el intervalo de confianza extremadamente amplio que se estimó. Sería pues deseable poder disponer de datos más contundentes acerca de la potencial mejora que implica el uso de BICI en pacientes con DM1 en el control de la $\mathrm{HbA}_{1}$ con el fin de poder tener unas estimaciones más consistentes.

Es importante señalar que las características basales y los factores de riesgo de la cohorte de pacientes que se simuló en el modelo han sido obtenidos a partir de una muestra del registro de pacientes con BICI de Cataluña ${ }^{20}$.

Cabe señalar otra limitación con la que nos hemos encontrado a la hora de llevar a cabo la adaptación del modelo para el ámbito español, y es que en algunos de los submodelos de los que está compuesto, como es el caso de las patologías de carácter cardiovascular, se ha usado la ecuación de riesgo de Framingham para evaluar este tipo de complicaciones. Existe cierta controversia sobre el uso de dicha ecuación para la población española. Ciertos estudios han demostrado que sobreestima el riesgo de eventos cardiovasculares en la población española ${ }^{31-33}$, sin embargo hasta la fecha de la elaboración del presente estudio no se ha llevado a cabo ninguna validación de esta ecuación para nuestro país. Los estudios que se han publicado 
han sido sin base poblacional, con un número y un diseño insuficientes como para poder tomar los resultados como extrapolables a la población española. Sin embargo, a falta de otra es importante mencionar que dicha ecuación de riesgo ha sido empleada en varios estudios llevados a cabo en el ámbito español para estimar el riesgo cardiovascular, tanto en estudios epidemiológicos ${ }^{34-37}$ como en diferentes evaluaciones económicas $^{38-40}$. Nuestra opinión es que dicha limitación no debería afectar de forma significativa los resultados de nuestro estudio, debido a que se ha usado esta ecuación en ambas ramas del modelo, por lo que la supuesta sobreestimación de eventos se ha dado por igual para ambos tratamientos.

En el modelo sólo se tuvieron en cuenta los costes directos sanitarios y no se pudieron incluir los costes indirectos, debido principalmente a la falta de datos. Se debe señalar que en los pacientes con DM1 los costes asociados a la falta de productividad laboral así como a la pérdida de días de trabajo o los costes de transporte, comprenden gran parte de este coste que desde el punto de vista social es muy importante. El uso de BICI mejora el control glucémico y beneficia con una reducción de acontecimientos adversos importantes, por lo que es de suponer que la inclusión de este tipo de costes en nuestro estudio no debería incrementar el valor del coste incremental por AVAC.

El análisis coste-utilidad mostró un resultado de $29.947 €$ GAVAC, lo que sitúa el uso de BICI frente a DMI como una terapia eficiente en el ámbito español ${ }^{41}$, siempre que el valor de la disposición a pagar por AVAC sea inferior a esta cifra. El uso de BICI no está indicado para cualquier paciente con DM1, sino que ha de cumplir ciertas condiciones que se encuentran muy bien definidas a nivel legal, por lo que los resultados de nuestro estudio podrían ser aplicables a una población de pacientes muy concreta, como la que se ha tenido en cuenta en este análisis ${ }^{20}$.
Sería conveniente disponer de datos de costes indirectos para el ámbito español con el fin de poder contemplarlos en el modelo. Pensamos que de esa forma quedaría todavía más reforzado desde un punto de vista económico, el uso de BICI frente a MDI.

\section{BIBLIOGRAFÍA}

1. Ettaro L, Songer TJ, Zhang P, Engelgau MM: Costof-illness studies in diabetes mellitus. Pharmacoeconomics 2004; 22:149-64.

2. Goday A: Epidemiología de la diabetes y sus complicaciones no coronarias. Rev.Esp.Cardiol 2002; 55:657-670.

3. Goday A, Serrano-Rios M: Epidemiología de la Diabetes Mellitus en España. Revisión crítica y nuevas perspectivas. Med.Clin.(Barc.) 1994; 102:306-15.

4. Bode BW, Sabbah HT, Gross TM, Fredrickson LP, Davidson PC: Diabetes management in the new millennium using insulin pump therapy. Diabetes Metab Res.Rev 2002;18 Suppl 1:S14-S20.

5. Lenhard MJ, Reeves GD: Continuous subcutaneous insulin infusion: a comprehensive review of insulin pump therapy. Arch.Intern.Med. 2001; 161:2293-300.

6. Pickup J, Mattock M, Kerry S: Glycaemic control with continuous subcutaneous insulin infusion compared with intensive insulin injections in patients with type 1 diabetes: meta-analysis of randomised controlled trials. BMJ 2002; 324:705.

7. Weissberg-Benchell J, Antisdel-Lomaglio J, Seshadri R: Insulin pump therapy: a meta-analysis. Diabetes Care 2003; 26:1079-87.

8. Weinstein MC, O'Brien B, Hornberger J, Jackson J, Johannesson M, McCabe C, Luce BR: Principles of good practice for decision analytic modeling in health-care evaluation: report of the ISPOR Task Force on Good Research Practices-Modeling Studies. Value.Health 2003; 6:9-17.

9. Rodriguez Barrios JM: El papel de los modelos en la evaluación económica en el campo sanitario. Farm.Hosp 2004; 28:231-42.

10. Malone DC: The role of pharmacoeconomic modeling in evidence-based and value-based formulary guidelines. J.Manag.Care Pharm 2005; 11:S7-10. 
11. Palmer AJ, Roze S, Valentine WJ, Minshall ME, Foos V, Lurati FM, Lammert M, Spinas GA: The CORE Diabetes Model: Projecting long-term clinical outcomes, costs and cost-effectiveness of interventions in diabetes mellitus (types 1 and 2) to support clinical and reimbursement decision-making. Curr.Med.Res.Opin 2004; 20 Suppl 1:S5-26.

12. Palmer AJ, Roze S, Valentine WJ, Minshall ME, Foos V, Lurati FM, Lammert M, Spinas GA: Validation of the CORE Diabetes Model against epidemiological and clinical studies. Curr.Med.Res. Opin. 2004; 20 Suppl 1:S27-S40.

13. D'Agostino RB, Russell MW, Huse DM, Ellison RC, Silbershatz H, Wilson PW, Hartz SC: Primary and subsequent coronary risk appraisal: new results from the Framingham study. Am.Heart J 2000; 139:272-81.

14. The Diabetes Control and Complications Trial Research Group. The effect of intensive diabetes therapy on the development and progression of neuropathy. Ann.Intern.Med 1995; 122:561-8.

15. UK Prospective Diabetes Study Group. Tight blood pressure control and risk of macrovascular and microvascular complications in type 2 diabetes: UKPDS 38. BMJ 1998; 17:703-13.

16. Adler AI, Stratton IM, Neil HA, Yudkin JS, Matthews DR, Cull CA, Wright AD, Turner RC, Holman RR: Association of systolic blood pressure with macrovascular and microvascular complications of type 2 diabetes (UKPDS 36): prospective observational study. BMJ 2000; 321:412-9.

17. Janghorbani MB, Jones RB, Allison SP: Incidence of and risk factors for cataract among diabetes clinic attenders. Ophthalmic Epidemiol 2000; 7:13-25.

18. The Diabetes Control and Complications Trial Research Group.The effect of intensive treatment of diabetes on the development and progression of long-term complications in insulin-dependent diabetes mellitus. N.Engl.J.Med 1993; 329:977-86.

19. Effect of intensive therapy on the microvascular complications of type 1 diabetes mellitus. JAMA 2002;87:2563-9.

20. Canton A, Fernandez CM, Conget I, Carreras G, Castell C, Tresserras R: Type 1 diabetes mellitus in Catalonia: chronic complications and metabolic control ten years after onset. Med.Sci.Monit 2004; 10:CR185-CR190.

21. Instituto Nacional de Estadística. Actualizado según el IPC de los productos sanitarios. Disponible en: http://www.ine.es . Madrid: INE; 2005.
22. Desgagne A, Castilloux AM, Angers JF, LeLorier $\mathrm{J}$ : The use of the bootstrap statistical method for the pharmacoeconomic cost analysis of skewed data. Pharmacoeconomics 1998; 13:487-97.

23. The Diabetes Control and Complications Trial Research Group.The effect of intensive treatment of diabetes on the development and progression of long-term complications in insulin-dependent diabetes mellitus. N.Engl.J.Med. 1993;29:977-86.

24. Roel P.L.M Hoogma: Disponible en: http: //www.diabetes-symposium.org/index.php? menu $=$ lectures $\&$ archive $=0 \&$ topicid $=15$. Citadoel 02/04/2005.

25. Bode BW, Sabbah HT, Gross TM, Fredrickson LP, Davidson PC: Diabetes management in the new millennium using insulin pump therapy. Diabetes Metab Res.Rev 2002; 18 Suppl 1:S14-S20.

26. Pickup J, Mattock M, Kerry S: Glycaemic control with continuous subcutaneous insulin infusion compared with intensive insulin injections in patients with type 1 diabetes: meta-analysis of randomised controlled trials. BMJ 2002; 324:705.

27. Linkeschova R, Raoul M, Bott U, Berger M, Spraul M: Less severe hypoglycaemia, better metabolic control, and improved quality of life in Type 1 diabetes mellitus with continuous subcutaneous insulin infusion (CSII) therapy; an observational study of 100 consecutive patients followed for a mean of 2 years. Diabet.Med 2002; 19:746-51.

28. DeWitt DE, Hirsch IB: Outpatient insulin therapy in type 1 and type 2 diabetes mellitus: scientific review. JAMA 2003; 289:2254-64.

29. National Institute of Clinical Excellence. Guide to the methods of technology appraisal. Londres: NICE; 2004.

30. Bruttomesso D, Pianta A, Crazzolara D, Scaldaferri E, Lora L, Guarneri G, Mongillo A, Gennaro R, Miola M, Moretti M, Confortin L, Beltramello GP, Pais M, Baritussio A, Casiglia E, Tiengo A: Continuous subcutaneous insulin infusion (CSII) in the Veneto region: efficacy, acceptability and quality of life. Diabet.Med 2002; 19:628-34.

31. Costa B, Cabre J, Martin F, Pinol J, Basora J, Blade $\mathrm{J}$ : La escala de Framingham sobrevalora el riesgo cerebrovascular de la diabetes y el síndrome metabólico de la población española. Aten.Primaria 2005; 35:392-8.

32. Ramos R, Solanas P, Cordon F, Rohlfs I, Elosua R, Sala J, Masia R, Faixedas MT, Marrugat J: Compa- 
ración de la función de Framingham original y la calibrada del REGICOR en la predicción del riesgo coronario poblacional. Med.Clin.(Barc.) 2003; 121:521-6.

33. Marrugat J, D’Agostino R, Sullivan L, Elosua R, Wilson P, Ordovas J, Solanas P, Cordon F, Ramos R, Sala J, Masia R, Kannel WB: An adaptation of the Framingham coronary heart disease risk function to European Mediterranean areas. J.Epidemiol.Community Health 2003; 57:634-8.

34. de la Pena FA, Suarez FC, Cuende M, I, Munoz RM, Garre CJ, Camafort BM, Roca VB, Alcala PJ: Control integral de los factores de riesgo en pacientes de alto y muy alto riesgo cardiovascular en España. Estudio CIFARC. Med.Clin.(Barc.) 2005; 124:44-9.

35. Aranceta J, Perez RC, Foz SM, Mantilla T, Serra ML, Moreno B, Monereo S, Millan J: Tablas de evaluación del riesgo coronario adaptadas a la población española: estudio DORICA. Med. Clin.(Barc.) 2004; 123:686-91.

36. Banegas B, Jr., Rodriguez-Artalejo F, de la Cruz Troca JJ, de Andres MB, del Rey CJ: Mortalidad relacionada con la hipertensión y la presión arterial en España. Med.Clin.(Barc.) 1999; 112:489-94.

37. Otzet I, Costa B, Franch J, Morato J, Pons P: [Cardiovascular risk and glucose metabolism: agreements and discrepancies between the WHO-85 and ADA-97 classifications]. Aten.Primaria 2002; 29:205-12.

38. Latour-Perez J, Navarro-Ruiz A, Ridao-Lopez M, Cervera-Montes M: Using clopidogrel in non-STsegment elevation acute coronary syndrome patients: a cost-utility analysis in Spain. Value. Health 2004; 7:52-60.

39. Plans RP, Rovira FJ: Estudio coste-efectividad de los tratamientos farmacológicos hipolipemiantes. Med.Clin.(Barc.) 1995;105:327-33.

40. Rubio-Terres C, Rodriguez J, Bolinder B, de Pablos P: Cost-Utility Analysis of Insulin Glargine Comapred with NPH Insulin in Patients with Type 1 Diabetes in Spain. Diabetes 2004; 53.

41. Sacristan JA, Oliva J, Del Llano J, Prieto L, Pinto JL: ¿Qué es una tecnología sanitaria eficiente en España? Gac.Sanit 2002; 16:334-43.

42. Clarke P, Gray A, Holman R: Estimating utility values for health states of type 2 diabetic patients using the EQ-5D (UKPDS 62). Med.Decis.Making $2002 ; 22: 340-9$.
43. Mathers CD, Vos ET, Stevenson CE, Begg SJ. The Burden of Disease and Injury in Australia. Bull World Health Organ. 2001;79(11):1076-84.

44. Tengs TO, Wallace A: One thousand health-related quality-of-life estimates. Med.Care 2000; 38:583637.

45. Carrington AL MSMMKJBAJP: Psychological status of diabetic people with or without lower limb disability. Diabetes Res Clin Pract 1996; 32:19-25.

46. NICE. NICE National Institute for Clinical Excellence. Technology appraisal guidance - No. 53. Guidance on the use of long-acting insuiln analogues for the treatment of diabetes - insulin glargine. 1-12-2002. 2006,

47. Levy E, Gabriel S, Dinet J: The comparative medical costs of atherothrombotic disease in European countries. Pharmacoeconomics 2003; 21:651-9.

48. Oliva J, Lobo F, Molina B, Monereo S: Direct health care costs of diabetic patients in Spain. Diabetes Care 2004; 2616-21.

49. Aros F, Loma-Osorio A, Alonso A, Alonso JJ, Cabades A, Coma-Canella I, Garcia-Castrillo L, Garcia E, Lopez dS, Pabon P, San Jose JM, Vera A, Worner F: Guías de actuación clínica de la Sociedad Española de Cardiología en el infarto agudo de miocardio. Rev.Esp.Cardiol 1999; 52:919-56.

50. Antoñanzas F, Anton F, Echevarria I, Juarez C: Clinica y Epidemiologia de la Insuficiencia Cardiaca Congestiva (ICC): Un analisis de Costes. Clinica cardiovascular 1998; 16:27-34.

51. Lamas J, Alonso M, Saavedra J, Garcia-Trio G, Rionda M, Ameijeiras M: Costes de la diálisis crónica en un hospital. público: mitos y realidades. Nefrologia. 2001; 21:283-94.

52. Real Decreto $1247 / 2002$ de 3 de diciembre por el que se regula la gestion del fondo de cohesion sanitaria. BOE núm 290: 04/12/2004.

53. De Alvaro F, Palmer AJ, Annemans L, Roze S, Lamotte M, Rodby RA. Coste-efectividad de irbesartan en pacientes hipertensos con nefropatía diabética tipo II: una perspectiva española. Nefrología 2004; 23:231-8.

54. Instituto de Salud Carlos III: Analisis coste-efectividad de diferentes estrategias para el cribado y tratamiento de la retinopatia diabetica en pacientes con diabetes mellitus. Madrid: Ministerio de Sanidad y onsumo; 2004. 
55. Castells X, Alonso J, Castilla M, Ribo C, Cots F, Anto JM: Outcomes and costs of outpatient and inpatient cataract surgery: a randomised clinical trial. J.Clin.Epidemiol 2001; 54:23-9.

56. Ghatnekar O, Persson U, Willis M, Odegaard K: Cost effectiveness of Becaplermin in the treatment of diabetic foot ulcers in four European countries. Pharmacoeconomics 2001; 19:767-78.
57. Hart WM, Espinosa C, Rovira J: A simulation model of the cost of the incidence of IDDM in Spain. Diabetologia 1997; 40:311-8.

58. Curtis JR, To T, Muirhead S, Cummings E, Daneman D: Recent trends in hospitalization for diabetic ketoacidosis in ontario children. Diabetes Care 2002; 25:1591-6. 